\title{
Radial transport in a porous medium with Dirichlet, Neumann and Robin-type inhomogeneous boundary values and general initial data: analytical solution and evaluation
}

\author{
E. J. M. Veling
}

Received: 7 February 2011 / Accepted: 9 September 2011 / Published online: 1 November 2011

(C) The Author(s) 2011. This article is published with open access at Springerlink.com

\begin{abstract}
The analytical solution is presented to the convection-diffusion equation describing the concentration of solutes in a radial velocity field due to extracting groundwater from or injecting water into an aquifer with arbitrary initial concentration data $F(r)$, with $r$ the radial distance, and an inhomogeneous mixed boundary condition $G(t)$, with $t$ the time, at the well radius $r=r_{0}$. The analytical solution is obtained with a generalized Hankel transformation or with a Laplace transformation. The Hankel transformation turns out to be easier for $G=0, F \neq 0$, while the Laplace transformation is easier for $F=0, G \neq 0$. Both techniques can, however, deal with the full problem. The representation found by the generalized Hankel transform can also be found by the Laplace transform, through modification of the contour through the complex plane in the Bromwich integral for the inverse Laplace transform to the real axis. In practice, the numerical evaluation of the integral representation is difficult, due to the oscillating behavior of the integrands. A more appropriate numerical inversion procedure is also suggested, which circumvents the integration of the oscillating integrands, by an alternative modification of the contour in the Bromwich integral such that the new contour follows the steepest descent path starting from a saddle point at the real axis.
\end{abstract}

Keywords Airy function - Analytical solution - Convection-diffusion · Initial-boundary value problem . Mixed boundary condition $\cdot$ Radial flow field $\cdot$ Steepest descent path

\section{Introduction}

Various authors have studied the convection-diffusion equation in a radial flow field. Approximate solutions were given by Hoopes and Harleman [1], Dagan [2], Gelhar and Collins [3], Bear [4, pp. 637-640], and Bachmat et al. [5]. Bear [4] gives a summary of previous work. Pure analytical approaches were presented by Ogata [6], Bear [4, pp. 635-637], Tang and Babu [7], Moench and Ogata [8], Hsieh [9], Chen [10], Chen and Woodside [11], Chen et al. [12], and Bruggeman [13]. Huang et al. [14] discuss the problem of a single-well push-pull tracer test taking into account the $z$-dependence and the solid phase. Directly related work was published by Chen [15], Chen [16], Valocchi [17], Moench [18], Yates [19], Goltz and Oxley [20], Moench [21], Chen et al. [22], Chen et al. [23], and Chen [24]. The paper by Chen et al. [25] studies a problem on a finite domain. The paper by Chen et al. [26] studies

E. J. M. Veling $(\bowtie)$

Faculty of Civil Engineering and Geosciences, Water Resources Section, Delft University of Technology, P.O. Box 5048, 2600 GA Delft, The Netherlands

e-mail: E.J.M.Veling@TUDelft.nl 
a problem without axial symmetry. The two last papers construct power series solutions. The paper by Leijnse et al. [27] published results somewhat restricted as presented below. Their work was based on mathematical results by M. Kopáčková published in an internal report [28].

In this article, we derive the analytical solution of the convection-diffusion equation in a radial flow field in full generality with respect to the initial condition and an inhomogeneous mixed boundary condition at the well radius. The mathematical expression of the solution of this problem has been published before in a conference proceedings [29]. In this article, the derivation of this expression is given, together with a discussion of how one can solve initial-boundary value problems using the Laplace transform with respect to the time variable and a generalized Hankel transform with respect to the spatial variable. Application of the Laplace transform requires a number of quite complicated mathematical manipulations to find the solution in the time domain, while application of the generalized Hankel transform delivers the solution much more easily, once the corresponding Hankel inversion formula has been found. We show how this Hankel inversion formula can be found and discuss the general technique for finding appropriate transforms and their inverses with respect to the spatial variable.

Numerical evaluation of the presented analytical expression is quite difficult. It is possible, however, by using appropriate numerical techniques. We compare the results of three different techniques. The first one is to evaluate the analytical solution numerically by performing the integrations. For the other two, we specify first the Laplace transform of the solution and apply two different numerical Laplace inversions. One is nowadays quite popular and very reliable: the method by de Hoog et al. [30]. The other method is the application of the steepest descent path through the complex $p$-plane (with $p$ the Laplace variable). One has easier control over the precision in this last method. Comparable methods have been discussed by Talbot [31], Evans [32], and Evans and Chung [33]. We found agreement between these three methods (direct explicit numerical integration, Laplace inversion by the method of de Hoog et al. [30] and Laplace inversion by the method of the steepest descent path) up to the required numerical precision.

\section{Formulation analytical solution}

At a well groundwater is extracted from or water is pumped into an aquifer (a horizontal layer below the surface, made of porous material). We study the physical problem of the concentration $c$ of a solute as function of the time $t$ and the radial distance $r$ to the center of the well. Groundwater can be contaminated by such a solute in a natural way or man-made. So, in this article, we solve the following problem (see Hoopes and Harleman [1] or Tang and Babu [7] for a derivation)

$$
\begin{aligned}
& \frac{\partial c}{\partial t}=a\left|v_{r}\right| \frac{\partial^{2} c}{\partial r^{2}}-v_{r} \frac{\partial c}{\partial r}, \quad r>r_{0}, t>0, v_{r}=A / r, \\
& \left.\left(\gamma^{\prime} c+\delta^{\prime} \frac{\partial c}{\partial r}\right)\right|_{\left(r_{0}, t\right)}=G(t), r=r_{0}, t>0, \\
& \left.c\right|_{(r, 0)}=F(r), \quad r \geq r_{0}, t=0,
\end{aligned}
$$

with the usual requirement of finite values of $c$ for $r \rightarrow \infty$. Here, $c\left[\mathrm{ML}^{-3}\right]$ denotes the concentration, $t$ [T] the time, $r[\mathrm{~L}]$ the radial distance to the center of the well, $a[\mathrm{~L}]$ the dispersivity, $v_{r}\left[\mathrm{LT}^{-1}\right]$ the radial velocity, and $\gamma^{\prime}[-]$ and $\delta^{\prime}[\mathrm{L}]$ coefficients to specify the inhomogeneous mixed boundary data $G(t)\left[\mathrm{ML}^{-3}\right]$, and $F(r)\left[\mathrm{ML}^{-3}\right]$ denotes the initial condition. Furthermore

$v_{r}=A / r, \quad A=Q /\left(2 \pi H n_{\mathrm{e}}\right)\left[\mathrm{L}^{2} \mathrm{~T}^{-1}\right]$,

with $Q\left[\mathrm{~L}^{3} \mathrm{~T}^{-1}\right]$ the recharge, $H[\mathrm{~L}]$ the thickness of the aquifer, and $n_{e}[-]$ the effective porosity. For an injection $(Q>0)$, we have $v_{r}>0$, and the opposite for an extraction. If one places at the well a condition on the so-called flux concentration (see Kreft and Zuber [34]) $c_{F}=c-D_{r} / v_{r} \frac{\partial c}{\partial r}$, with $D_{r}=a\left|v_{r}\right|\left[\mathrm{L}^{2} \mathrm{~T}^{-1}\right]$ the dispersion 
coefficient, one has $c_{\mathrm{F}}=c-a\left|v_{r}\right| / v_{r} \frac{\partial c}{\partial r}=c-a \operatorname{sign}(Q) \frac{\partial c}{\partial r}$. This means for an injection: $\gamma^{\prime}=1, \delta^{\prime}=-a$, and for an extraction: $\gamma^{\prime}=1, \delta^{\prime}=a$.

Solutions for (1) have been presented for different boundary and initial conditions:

1. Dirichlet condition at the well:

$\gamma^{\prime}=1, \delta^{\prime}=0$, and $G(t)=c_{0}, F(r)=0$ :

Ogata [6], Bear [4, pp. 635-637], Tang and Babu [7], Moench and Ogata [8], Hsieh [9], Beneš et al. [28], Leijnse et al. [27], Bruggeman [13, formula 820.01].

2. Mixed boundary condition at the well corresponding to an injection (flux concentration):

$\gamma^{\prime}=1, \delta^{\prime}=-a$, and $G(t)=c_{0}, F(r)=0$ :

Chen [10] (only the Laplace transform), Huang et al. [14] (only the Laplace transform and the Fourier transform for a model with $z$-dependence and an extra ordinary differential equation for the solid phase).

3. Mixed boundary condition at the well corresponding to an injection (flux concentration):

$\gamma^{\prime}=1, \delta^{\prime}=-a$, and $G(t)=\delta(t)$, the Dirac delta function, $F(r)=0, r_{0}=0$ :

Bruggeman [13, formula 820.02].

4. Neumann condition at the well, extraction:

$\gamma^{\prime}=0, \delta^{\prime}=a$, and $G(t)=0, F(r) \neq 0$ :

Beneš et al. [28], Leijnse et al. [27], Chen and Woodside [11], Chen et al. [12] (only the Laplace transform), Huang et al. [14] (only the Laplace transform and the Fourier transform for a model with $z$-dependence and an extra ordinary differential equation for the solid phase).

Here we shall give the general solution without any restrictions on $F(r), G(t), \gamma^{\prime}$, and $\delta^{\prime}$. By means of the following transformations:

$\tau=\left(|A| / a^{2}\right) t, \quad \rho=r / a, \quad \rho_{0}=r_{0} / a$,

the problem becomes dimensionless:

$$
\begin{aligned}
& \frac{\partial C}{\partial \tau}=\frac{1}{\rho}\left(\frac{\partial^{2} C}{\partial \rho^{2}} \pm \frac{\partial C}{\partial \rho}\right), \quad \rho>\rho_{0}, \tau>0, \\
& \left.\left(\gamma C+\delta \frac{\partial C}{\partial \rho}\right)\right|_{\left(\rho_{0}, \tau\right)}=g(\tau), \rho=\rho_{0}, \tau>0, \\
& \left.C\right|_{(\rho, 0)}=f(\rho), \quad \rho \geq \rho_{0}, \tau=0,
\end{aligned}
$$

with $C=c / G_{0}, \gamma=\gamma^{\prime}, \delta=\delta^{\prime} / a, g(\tau)=G(t) / G_{0}, f(\rho)=F(r) / G_{0}$, and $G_{0}\left[\mathrm{ML}^{-3}\right]$ a normalization constant. The upper (plus) sign represents an extraction $(Q<0)$, the lower (minus) sign an injection $(Q>0)$. The analytical solution of the problem defined by (4) has been given by Eq. 5 in Veling [29] without derivation, and is expressed in Airy functions (see Chap. 10.4 in Abramowitz and Stegun [35]) as

$$
\begin{aligned}
C(\rho, \tau)= & \mathrm{e}^{\mp \rho / 2} \int_{0}^{\infty}\left\{s^{-1 / 3} \frac{A i(z) B_{0}\left(z_{0}\right)-B i(z) A_{0}\left(z_{0}\right)}{A_{0}^{2}\left(z_{0}\right)+B_{0}^{2}\left(z_{0}\right)} \mathrm{e}^{-s \tau}\right. \\
& \left.\times \int_{\rho_{0}}^{\infty} \rho^{\prime} \mathrm{e}^{ \pm \rho^{\prime} / 2} f\left(\rho^{\prime}\right)\left\{A i\left(z^{\prime}\right) B_{0}\left(z_{0}\right)-B i\left(z^{\prime}\right) A_{0}\left(z_{0}\right)\right\} \mathrm{d} \rho^{\prime}\right\} \mathrm{d} s \\
& +\mathrm{e}^{\mp\left(\rho-\rho_{0}\right) / 2} \int_{0}^{\infty}\left\{\frac{A i(z) B_{0}\left(z_{0}\right)-B i(z) A_{0}\left(z_{0}\right)}{\pi\left(A_{0}^{2}\left(z_{0}\right)+B_{0}^{2}\left(z_{0}\right)\right)} \int_{0}^{\tau} g\left(\tau-\tau^{\prime}\right) \mathrm{e}^{-s \tau^{\prime}} \mathrm{d} \tau^{\prime}\right\} \mathrm{d} s,
\end{aligned}
$$


with

$$
\begin{aligned}
& z^{\prime}=\frac{1-4 s \rho^{\prime}}{4 s^{2 / 3}}, \quad z_{0}=\frac{1-4 s \rho_{0}}{4 s^{2 / 3}}, \quad z=\frac{1-4 s \rho}{4 s^{2 / 3}}, \\
& A_{0}\left(z_{0}\right)=\left(\gamma \mp \frac{1}{2} \delta\right) A i\left(z_{0}\right)-\delta s^{1 / 3} A i^{\prime}\left(z_{0}\right), \quad B_{0}\left(z_{0}\right)=\left(\gamma \mp \frac{1}{2} \delta\right) B i\left(z_{0}\right)-\delta s^{1 / 3} B i^{\prime}\left(z_{0}\right) .
\end{aligned}
$$

Specifically, for Dirichlet boundary data, we have:

$$
\begin{aligned}
& \gamma^{\prime}=1, \delta^{\prime}=0, \text { so } \gamma=1, \delta=0, \\
& A_{0}\left(z_{0}\right)=A i\left(z_{0}\right), B_{0}\left(z_{0}\right)=B i\left(z_{0}\right),
\end{aligned}
$$

for Neumann boundary data, see also Chen and Woodside [11], and Chen et al. [12]:

$$
\begin{aligned}
& \gamma^{\prime}=0, \delta^{\prime}= \pm a, \text { so } \gamma=0, \delta= \pm 1, \\
& A_{0}\left(z_{0}\right)=-\frac{1}{2} A i\left(z_{0}\right) \mp s^{1 / 3} A i^{\prime}\left(z_{0}\right), \quad B_{0}\left(z_{0}\right)=-\frac{1}{2} B i\left(z_{0}\right) \mp s^{1 / 3} B i^{\prime}\left(z_{0}\right),
\end{aligned}
$$

and for mixed boundary data, see also Chen [10]:

$$
\begin{aligned}
& \gamma^{\prime}=1, \delta^{\prime}= \pm a, \text { so } \gamma=1, \delta= \pm 1, \\
& A_{0}\left(z_{0}\right)=\frac{1}{2} A i\left(z_{0}\right) \mp s^{1 / 3} A i^{\prime}\left(z_{0}\right), \quad B_{0}\left(z_{0}\right)=\frac{1}{2} B i\left(z_{0}\right) \mp s^{1 / 3} B i^{\prime}\left(z_{0}\right) .
\end{aligned}
$$

At first sight, the result (5) deviates from formula (36) in Chen and Woodside [11], under the restrictions used by these authors. However, by applying some elementary algebra their solution can be put in the more concise form (5). Some authors do not use the presentation of the analytical solution in terms of Airy functions (e.g., Tang and Babu [7], Beneš et al. [28]), but they use Bessel functions with index 1/3. Their expressions are somewhat more complicated.

\section{Generalized Hankel transform}

In this section, we derive a generalized Hankel transform. First, the convection term will be removed by means of the substitution

$C(\rho, \tau)=D(\rho, \tau) \mathrm{e}^{\mp \rho / 2}$,

then (4) becomes

$$
\begin{array}{ll}
\frac{\partial D}{\partial \tau}=\frac{1}{\rho}\left(\frac{\partial^{2} D}{\partial \rho^{2}}-\frac{1}{4} D\right), & \rho>\rho_{0}, \tau>0, \\
\left.\left(\alpha D+\beta \frac{\partial D}{\partial \rho}\right)\right|_{\left(\rho_{0}, \tau\right)}=\mathrm{e}^{ \pm \rho_{0} / 2} g(\tau), \rho=\rho_{0}, \tau>0, & \rho \geq \rho_{0}, \tau=0,
\end{array}
$$

with $\alpha=\gamma \mp \frac{1}{2} \delta, \beta=\delta$.

Next, we will use a form of Hankel transform related to the spatial variable, in contrast to the usual Laplace transform technique related to the time variable. Using the theory given in $\S \S 21.3,21.4,21.5$ in Naimark [36], it can be derived that for the following transformation

$$
\phi(s)=T[f] \equiv \int_{\rho_{0}}^{\infty} \rho f(\rho)\left\{\beta D_{1}(\rho, s)+\alpha D_{2}(\rho, s)\right\} \mathrm{d} \rho,
$$

with

$$
\begin{aligned}
& D_{1}(\rho, s)=\pi\left(A i(z) B i^{\prime}\left(z_{0}\right)-B i(z) A i^{\prime}\left(z_{0}\right)\right), \quad D_{2}(\rho, s)=-\pi s^{-1 / 3}\left(A i(z) B i\left(z_{0}\right)-B i(z) A i\left(z_{0}\right)\right), \\
& z_{0}=\frac{1-4 s \rho_{0}}{4 s^{2 / 3}}, \quad z=\frac{1-4 s \rho}{4 s^{2 / 3}},
\end{aligned}
$$


the back-transformation reads (see Appendix A)

$f(\rho)=T^{-1}[\phi]=\int_{0}^{\infty} \phi(s) \frac{\beta D_{1}(\rho, s)+\alpha D_{2}(\rho, s)}{\pi^{2}\left(A_{0}^{2}\left(z_{0}\right)+B_{0}^{2}\left(z_{0}\right)\right)} s^{1 / 3} \mathrm{~d} s$,

where $A_{0}\left(z_{0}\right)$ and $B_{0}\left(z_{0}\right)$ have been defined as (compare (6))

$A_{0}\left(z_{0}\right)=\alpha A i\left(z_{0}\right)-\beta s^{1 / 3} A i^{\prime}\left(z_{0}\right), \quad B_{0}\left(z_{0}\right)=\alpha B i\left(z_{0}\right)-\beta s^{1 / 3} B i^{\prime}\left(z_{0}\right)$.

Note that

$D_{1}\left(\rho_{0}, s\right)=1, \frac{\partial D_{1}}{\partial \rho}\left(\rho_{0}, s\right)=0$,

$D_{2}\left(\rho_{0}, s\right)=0, \frac{\partial D_{2}}{\partial \rho}\left(\rho_{0}, s\right)=-1$,

from the fact that $A i\left(z_{0}\right) B i^{\prime}\left(z_{0}\right)-B i\left(z_{0}\right) A i^{\prime}\left(z_{0}\right)=1 / \pi$, see formula (10.4.10) in Abramowitz and Stegun [35].

This Hankel transform has the operational property

$T\left[\frac{1}{\rho}\left(\frac{\partial^{2} f}{\partial \rho^{2}}-\frac{1}{4} f\right)\right]=-s T[f]$ if $f$ satisfies $\left.\left(\alpha f+\beta \frac{\partial f}{\partial \rho}\right)\right|_{\left(\rho=\rho_{0}\right)}=0$.

We remark that these transforms can also be written as

$$
\begin{aligned}
& \phi(s)=T[f]=\int_{\rho_{0}}^{\infty} \rho f(\rho)\left\{\left(-\pi s^{-1 / 3}\right)\left(A i(z) B_{0}\left(z_{0}\right)-B i(z) A_{0}\left(z_{0}\right)\right)\right\} \mathrm{d} \rho, \\
& f(\rho)=T^{-1}[\phi]=\int_{0}^{\infty} \phi(s) \frac{\left(-A i(z) B_{0}\left(z_{0}\right)+B i(z) A_{0}\left(z_{0}\right)\right)}{\pi\left(A_{0}^{2}\left(z_{0}\right)+B_{0}^{2}\left(z_{0}\right)\right)} \mathrm{d} s, \\
& z_{0}=\frac{1-4 s \rho_{0}}{4 s^{2 / 3}}, \quad z=\frac{1-4 s \rho}{4 s^{2 / 3}}
\end{aligned}
$$

since

$\beta D_{1}(\rho, s)+\alpha D_{2}(\rho, s)=\left(-\pi s^{-1 / 3}\right)\left(A i(z) B_{0}\left(z_{0}\right)-B i(z) A_{0}\left(z_{0}\right)\right)$.

If we accept the validity of these transforms for the moment, the derivation of the solution (5) reads as follows. First, write $D=D^{h}+D^{p}$, where $D^{h}$ is the solution of (11) with $g(\tau)=0$, and $D^{p}$ is a particular function which satisfies the inhomogeneous boundary condition at $\rho=\rho_{0}$. For $\alpha \neq \frac{1}{2} \beta$, let

$D^{p}(\rho, \tau)=\frac{g(\tau) \mathrm{e}^{ \pm \rho_{0} / 2+\rho_{0} / 2}}{\alpha-\frac{1}{2} \beta} \mathrm{e}^{-\rho / 2}$,

and for $\alpha=\frac{1}{2} \beta$

$D^{p}(\rho, \tau)=\frac{g(\tau) \mathrm{e}^{ \pm \rho_{0} / 2+\rho_{0} / 2}}{\beta} \rho \mathrm{e}^{-\rho / 2}$,

then $D^{p}$ satisfies

$$
\left.\left(\alpha D^{p}+\beta \frac{\partial D^{p}}{\partial \rho}\right)\right|_{\left(\rho_{0}, \tau\right)}=\mathrm{e}^{ \pm \rho_{0} / 2} g(\tau) .
$$

This implies that the function $D^{h}$ has to satisfy

$$
\begin{array}{ll}
\frac{\partial D^{h}}{\partial \tau}-\frac{1}{\rho}\left(\frac{\partial^{2} D^{h}}{\partial \rho^{2}}-\frac{1}{4} D^{h}\right)=-\frac{\partial D^{p}}{\partial \tau}+\frac{1}{\rho}\left(\frac{\partial^{2} D^{p}}{\partial \rho^{2}}-\frac{1}{4} D^{p}\right), & \rho>\rho_{0}, \tau>0, \\
\left.\left(\alpha D^{h}+\beta \frac{\partial D^{h}}{\partial \rho}\right)\right|_{\left(\rho_{0}, \tau\right)}=0, & \tau>0, \\
\left.D^{h}\right|_{(\rho, 0)}=\mathrm{e}^{ \pm \rho / 2} f(\rho)-D^{p}(\rho, 0), & \rho>\rho_{0} .
\end{array}
$$


We now apply the Hankel transform. Define $T\left[D^{h}\right]=\hat{D}(\tau ; s)$. The function $\hat{D}(\tau ; s)$ satisfies

$$
\begin{aligned}
& \frac{d \hat{D}}{d \tau}+s \hat{D}=-\frac{d T\left[D^{p}\right]}{d \tau}+T\left[\frac{1}{\rho}\left(\frac{\partial^{2} D^{p}}{\partial \rho^{2}}-\frac{1}{4} D^{p}\right)\right], \tau>0, \\
& \hat{D}(0 ; s)=T\left[\mathrm{e}^{ \pm \rho / 2} f(\rho)-D^{p}(\rho, 0)\right] \equiv \hat{D}_{0}(s) .
\end{aligned}
$$

First, we treat the case in which $\alpha \neq \frac{1}{2} \beta$. Then, the last term in the ordinary differential equation above cancels and by standard techniques it follows that

$$
\begin{aligned}
& \hat{D}(\tau ; s)=\left(\hat{D}_{0}(s)-\int_{0}^{\tau} \frac{d}{d \tau^{\prime}} T\left[D^{p}\right]\left(\tau^{\prime} ; s\right) \mathrm{e}^{s \tau^{\prime}} \mathrm{d} \tau^{\prime}\right) \mathrm{e}^{-s \tau} \\
& =T\left[\mathrm{e}^{ \pm \rho / 2} f(\rho)\right] \mathrm{e}^{-s \tau}-\frac{g(\tau) \mathrm{e}^{ \pm \rho_{0} / 2+\rho_{0} / 2}}{\alpha-\frac{1}{2} \beta} T\left[\mathrm{e}^{-\rho / 2}\right]+T\left[\mathrm{e}^{-\rho / 2}\right] \int_{0}^{\tau} \frac{g\left(\tau-\tau^{\prime}\right) \mathrm{e}^{ \pm \rho_{0} / 2+\rho_{0} / 2}}{\alpha-\frac{1}{2} \beta} s \mathrm{e}^{-s \tau^{\prime}} \mathrm{d} \tau^{\prime} .
\end{aligned}
$$

Applying the back-transformation, we find

$$
\begin{aligned}
& D(\rho, \tau)=T^{-1}[\hat{D}]+D^{p}(\rho, \tau) \\
& =T^{-1}\left[\mathrm{e}^{-s \tau} T\left[\mathrm{e}^{ \pm \rho / 2} f(\rho)\right]\right]+T^{-1}\left[T\left[\mathrm{e}^{-\rho / 2}\right] \int_{0}^{\tau} \frac{g\left(\tau-\tau^{\prime}\right) \mathrm{e}^{ \pm \rho_{0} / 2+\rho_{0} / 2}}{\alpha-\frac{1}{2} \beta} s \mathrm{e}^{-s \tau^{\prime}} \mathrm{d} \tau^{\prime}\right] .
\end{aligned}
$$

The term $D^{p}(\rho, \tau)$ cancels with the second term in the second line of (26). We denote

$R_{1}(\rho, \tau)=T^{-1}\left[\mathrm{e}^{-s \tau} T\left[\mathrm{e}^{ \pm \rho / 2} f(\rho)\right]\right]$

and

$R_{2}(\rho, \tau)=T^{-1}\left[T\left[\mathrm{e}^{-\rho / 2}\right] \int_{0}^{\tau} \frac{g\left(\tau-\tau^{\prime}\right) \mathrm{e}^{ \pm \rho_{0} / 2+\rho_{0} / 2}}{\alpha-\frac{1}{2} \beta} s \mathrm{e}^{-s \tau^{\prime}} \mathrm{d} \tau^{\prime}\right]$.

Now, $R_{1}(\rho, \tau)$ is written as

$$
\begin{aligned}
& R_{1}(\rho, \tau)=\int_{0}^{\infty}\left\{s^{-1 / 3} \frac{A i(z) B_{0}\left(z_{0}\right)-B i(z) A_{0}\left(z_{0}\right)}{A_{0}^{2}\left(z_{0}\right)+B_{0}^{2}\left(z_{0}\right)} \mathrm{e}^{-s \tau}\right. \\
& \left.\times \int_{\rho_{0}}^{\infty} \rho^{\prime} \mathrm{e}^{ \pm \rho^{\prime} / 2} f\left(\rho^{\prime}\right)\left\{A i\left(z^{\prime}\right) B_{0}\left(z_{0}\right)-B i\left(z^{\prime}\right) A_{0}\left(z_{0}\right)\right\} \mathrm{d} \rho^{\prime}\right\} \mathrm{d} s,
\end{aligned}
$$

and $R_{2}(\rho, \tau)$ as

$$
R_{2}(\rho, \tau)=-\int_{0}^{\infty}\left\{\frac{A i(z) B_{0}\left(z_{0}\right)-B i(z) A_{0}\left(z_{0}\right)}{\pi\left(A_{0}^{2}\left(z_{0}\right)+B_{0}^{2}\left(z_{0}\right)\right)} T\left[\mathrm{e}^{-\rho / 2}\right] \int_{0}^{\tau} \frac{g\left(\tau-\tau^{\prime}\right) \mathrm{e}^{ \pm \rho_{0} / 2+\rho_{0} / 2}}{\alpha-\frac{1}{2} \beta} s \mathrm{e}^{-s \tau^{\prime}} \mathrm{d} \tau^{\prime}\right\} \mathrm{d} s,
$$

with the aid of the identities (see (20))

$$
\begin{aligned}
& \beta D_{1}(\rho, s)+\alpha D_{2}(\rho, s)=\left(-\pi s^{-1 / 3}\right)\left(A i(z) B_{0}\left(z_{0}\right)-B i(z) A_{0}\left(z_{0}\right)\right), \\
& \beta D_{1}\left(\rho^{\prime}, s\right)+\alpha D_{2}\left(\rho^{\prime}, s\right)=\left(-\pi s^{-1 / 3}\right)\left(A i\left(z^{\prime}\right) B_{0}\left(z_{0}\right)-B i\left(z^{\prime}\right) A_{0}\left(z_{0}\right)\right) .
\end{aligned}
$$

The term $T\left[\mathrm{e}^{-\rho / 2}\right]$ remains to be evaluated, see Appendix B. There we find (see (71))

$$
T\left[\mathrm{e}^{-\rho / 2}\right]=-s^{-1} \mathrm{e}^{-\rho_{0} / 2}\left(\alpha-\frac{1}{2} \beta\right) .
$$


Insertion of (33) delivers for (31)

$R_{2}(\rho, \tau)=\mathrm{e}^{ \pm \rho_{0} / 2} \int_{0}^{\infty}\left\{\frac{A i(z) B_{0}\left(z_{0}\right)-B i(z) A_{0}\left(z_{0}\right)}{\pi\left(A_{0}^{2}\left(z_{0}\right)+B_{0}^{2}\left(z_{0}\right)\right)} \int_{0}^{\tau} g\left(\tau-\tau^{\prime}\right) \mathrm{e}^{-s \tau^{\prime}} \mathrm{d} \tau^{\prime}\right\} \mathrm{d} s$.

Putting the pieces (10), (30) and (34) together, we find the result (5).

In the case $\alpha=\frac{1}{2} \beta$, the derivation follows the same lines. However, on the right-hand side of the ordinary differential equation in (25) there is an extra term $-D^{p} / \rho^{2}$. The function $D$ in (27) therefore becomes

$$
\begin{aligned}
& D(\rho, \tau)=T^{-1}[\hat{D}]+D^{p}(\rho, \tau) \\
& =T^{-1}\left[\mathrm{e}^{-s \tau} T\left[\mathrm{e}^{ \pm \rho / 2} f(\rho)\right]\right]+T^{-1}\left[T\left[\rho \mathrm{e}^{-\rho / 2}-\frac{1}{\rho} s^{-1} \mathrm{e}^{-\rho / 2}\right] \int_{0}^{\tau} \frac{g\left(\tau-\tau^{\prime}\right) \mathrm{e}^{ \pm \rho_{0} / 2+\rho_{0} / 2}}{\beta} s \mathrm{e}^{-s \tau^{\prime}} \mathrm{d} \tau^{\prime}\right] .
\end{aligned}
$$

Then there remains the integral $T\left[\rho \mathrm{e}^{-\rho / 2}-\frac{1}{\rho} s^{-1} \mathrm{e}^{-\rho / 2}\right]$, see Appendix B. There we find (see (76))

$T\left[\rho \mathrm{e}^{-\rho / 2}-\frac{1}{\rho} s^{-1} \mathrm{e}^{-\rho / 2}\right]=-s^{-1} \mathrm{e}^{-\rho_{0} / 2} \beta$.

Putting together (10), (35) and (36) we find the same result as (5).

\section{Laplace transform}

Here, it will be sketched how one can arrive at the same result using the Laplace transform technique, defined as

$\mathcal{L}[f](p)=\tilde{f}(p)=\int_{0}^{\infty} \mathrm{e}^{-p t} f(t) \mathrm{d} t, \quad p \in \mathbb{C}$.

We start from (11). The function $\tilde{D}(\rho ; p)$ has to satisfy

$\frac{\mathrm{d}^{2} \tilde{D}}{\mathrm{~d} \rho^{2}}-\frac{1}{4} \tilde{D}=\rho\left(p \tilde{D}-\mathrm{e}^{ \pm \rho / 2} f(\rho)\right), \rho>\rho_{0}$,

$\left.\left(\alpha \tilde{D}+\beta \frac{\mathrm{d} \tilde{D}}{\mathrm{~d} \rho}\right)\right|_{\left(\rho_{0}, p\right)}=\mathrm{e}^{ \pm \rho_{0} / 2} \tilde{g}(p), \quad \rho=\rho_{0}$.

If one writes $\tilde{D}(\rho ; p)=\tilde{D}_{f=0}(\rho ; p)+\tilde{D}_{g=0}(\rho ; p)$, with $\tilde{D}_{f=0}(\rho ; p)$ the solution of $(38)$ if $f=0$, and $\tilde{D}_{g=0}(\rho ; p)$ the solution of (38) if $g=0$, then it is easy to find

$\tilde{D}_{f=0}(\rho ; p)=\frac{\mathrm{e}^{ \pm \rho_{0} / 2} \tilde{g}(p) A i(u)}{\bar{A}_{0}\left(u_{0}\right)}$,

with

$u=\frac{1+4 p \rho}{4 p^{2 / 3}}, \quad u_{0}=\frac{1+4 p \rho_{0}}{4 p^{2 / 3}}, \quad \bar{A}_{0}\left(u_{0}\right)=\alpha A i\left(u_{0}\right)+\beta p^{1 / 3} A i^{\prime}\left(u_{0}\right)$,

since the transformations in (40) transform (38) with $\tilde{D}(\rho ; p)=\check{D}(u ; p)$ into

$$
\begin{array}{ll}
\frac{\mathrm{d}^{2} \check{D}}{\mathrm{~d} u^{2}}=u \check{D}-\rho \mathrm{e}^{ \pm \rho / 2} f(\rho), & u>u_{0}, \\
\left.\left(\alpha \check{D}+\beta p^{1 / 3} \frac{\mathrm{d} \check{D}}{\mathrm{~d} u}\right)\right|_{\left(u_{0}, p\right)}=\mathrm{e}^{ \pm \rho_{0} / 2} \tilde{g}(p), u=u_{0},
\end{array}
$$


and since $A i(u)$ is the solution of $\frac{\mathrm{d}^{2} \check{D}}{\mathrm{~d} u^{2}}=u \check{D}$ with the correct behaviour at $u \rightarrow \infty$. The function $\tilde{D}_{g=0}(\rho ; p)$ is more difficult to find (by means of the method of variation of constants) as

$$
\begin{aligned}
& \tilde{D}_{g=0}(\rho ; p)=\frac{\pi}{\bar{A}_{0}\left(u_{0}\right)}\left\{\int_{\rho_{0}}^{\rho} \rho^{\prime} \mathrm{e}^{ \pm \rho^{\prime} / 2} f\left(\rho^{\prime}\right) p^{-1 / 3}\left\{A i(z)\left(\bar{A}_{0}\left(u_{0}\right) B i\left(z^{\prime}\right)-\bar{B}_{0}\left(u_{0}\right) A i\left(z^{\prime}\right)\right)\right\} \mathrm{d} \rho^{\prime}\right. \\
& \left.+\int_{\rho}^{\infty} \rho^{\prime} \mathrm{e}^{ \pm \rho^{\prime} / 2} f\left(\rho^{\prime}\right) p^{-1 / 3}\left\{A i\left(z^{\prime}\right)\left(\bar{A}_{0}\left(u_{0}\right) B i(z)-\bar{B}_{0}\left(u_{0}\right) A i(z)\right)\right\} \mathrm{d} \rho^{\prime}\right\},
\end{aligned}
$$

with additionally

$u^{\prime}=\frac{1+4 p \rho^{\prime}}{4 p^{2 / 3}}, \quad \bar{B}_{0}\left(u_{0}\right)=\alpha B i\left(u_{0}\right)+\beta p^{1 / 3} B i^{\prime}\left(u_{0}\right)$.

To find the analytical solution by inverting these transforms to the $\tau$-domain one has to deform the Bromwich contour (see Sect. 6, (45)) through the complex $p$-domain such that the singularity at $p=0$ is excluded. This will be done by integration along the negative real axis approaching from above and from below in the usual way and circling around $p=0$, clockwise. The calculations become quite cumbersome, but in the end one finds again (5).

\section{Discussion of transform techniques}

The advantage of the generalized Hankel transform is that it may be applied to find the solution of problem (4) in cases where $f \neq 0, g=0$ in a very easy way. Even when $g \neq 0$, and taking the validity of the generalized Hankel transform for granted, the derivation of the full solution is rather straightforward, albeit somewhat involved as shown above. When one applies the Laplace transform technique however, it turns out that the case $f \neq 0, g=0$ is the most complicated one. For the somewhat special case $\gamma^{\prime}=1, \delta^{\prime}=0$ (so $\gamma=1, \delta=0$ ) this has been performed by Chen and Woodside [11], see the remark in Sect. 2. But the derivation of the solution of problem (4) with $f=0, g \neq 0$ runs rather smoothly, although one has to do some calculations with Airy functions with complex arguments. Those kind of calculations have their counterpart in the derivation of the generalized Hankel transform itself.

Summarizing, the easiest way to solve problem (4) is to write the solution as $C=C_{f=0}+C_{g=0}$, where $C_{f=0}$ is the solution if $f=0$, and $C_{g=0}$ if $g=0 . C_{f=0}$ may be found easily by the Laplace transform, and $C_{g=0}$ by the generalized Hankel transform.

In general, this strategy applies to analogous problems. When one is interested in the closed form expression of the solution it might therefore be more advantageous to derive a transform with respect to the spatial variable in line with the generalized Hankel transform presented here (using the theory supplied in §§ 21.3, 21.4, 21.5 in Naimark [36] and Appendix A) than to apply the Laplace transform for all possible boundary and initial conditions.

In practical situations one also wants to evaluate the solution. The most efficient way to calculate (5) is to find the transformed solution and to perform a numerical inversion as explained in the next section. In any case, the closed form expression exhibits the dependency of the solution on the parameters.

\section{Numerical considerations}

Numerical evaluation of solution (5) yields quite a few problems, since the integrands exhibit oscillatory behaviour which can become a nightmare for the numerical analyst. See the discussion in Sect. 7.1. Numerical inversion of the Laplace transformed solution may be applied with the algorithms of the Stehfest method (see Stehfest [37], and Ogata and Moench [8]), the Talbot method (see Talbot [31], and Moench [18]) and, used most effectively in the past 25 years, the algorithm by de Hoog et al. [30], see also Moench [21]. For a unified framework for a number of Laplace inversion methods we refer to Abate and Whitt [38] and Avdis and Whitt [39]. 
Here, we present an alternative technique which performs the integration along the steepest descent path through the complex plane, see Chapter II.4, "Method of Steepest Descents" in Wong [40]. This method stems from theoretical work to derive asymptotic formulae for integrals. This method has received little attention, probably rooted in the fact that this method requires additional function evaluations. In the literature on Laplace inversion, comparable methods have been discussed by Talbot [31], Evans [32], and Evans and Chung [33]. These authors construct a suitable contour, although it is not the steepest descent path. For all methods which enter the complex plane, one has to evaluate the Laplace transformed solution for complex arguments and one needs thus complex versions of the mathematical functions. Moreover, all the calculations require complex arithmetic. Nowadays, complex versions for the standard mathematical functions are easily available, and for the Bessel-type functions one can exploit the impressive library of Amos (see [41-43]). This library includes the Airy-functions. Separate algorithms for real and complex Airy functions have been published by Gordon [44], and Schulten et al. [45], respectively.

The starting point to find the steepest descent path is the well-known Bromwich integral for the inversion of the Laplace transform. One writes the Laplace transform of $f$ as

$\mathcal{L}[f](p)=\tilde{f}(p)=\int_{0}^{\infty} \mathrm{e}^{-p t} f(t) \mathrm{d} t, \quad p \in \mathbb{C}$.

Depending on the properties of $f$, the function $\tilde{f}(p)$ exists for $\mathfrak{R e} p>p_{0}$ (e.g. if $f$ is piecewise continuous on $\left(0, t_{0}\right)$, for all $t_{0}>0$, and $|f(t)| \leq C \mathrm{e}^{\alpha t}$ for all $t>0$, and some $\left.C>0, \alpha>0\right)$. Then the Bromwich integral formula reads

$f(t)=\frac{1}{2 \pi \mathrm{i}} \int_{L} \mathrm{e}^{p t} \tilde{f}(p) \mathrm{d} p, t>0$,

where the contour $L$ is defined by

$L=\left\{p=p_{1}+\mathrm{i} q, p_{1}>p_{0}, p_{1}\right.$ fixed, $\left.q \in \mathbb{R}\right\}$.

Here, the contour $L$ will be deformed to the steepest descent path $L^{\prime}$ going through a saddle point $p=p_{0}$ at the real axis. This saddle point will be found by solving $\frac{\mathrm{d}}{\mathrm{d} p}\left(\mathrm{e}^{p t} \tilde{f}(p)\right)=0$. One of the two important properties of the steepest descent path $L^{\prime}$ is that along this contour Phase $\left(\mathrm{e}^{p t} \tilde{f}(p)\right)$ is constant. If Phase $\left(\mathrm{e}^{p t} \tilde{f}(p)\right)=0$, for $p=p_{0} \in \mathbf{R}$, which is in general the case, then Phase $\left(\mathrm{e}^{p t} \tilde{f}(p)\right)=0$, for all $p \in L^{\prime}$. This means that the integrand $\mathrm{e}^{p t} \tilde{f}(p)$ along $L^{\prime}$ stays real and positive, which is an important numerical advantage. The other property of the steepest descent path is that along $L^{\prime}\left|\mathrm{e}^{p t} \tilde{f}(p)\right|\left(=\mathfrak{R e}\left(\mathrm{e}^{p t} \tilde{f}(p)\right)\right.$ decreases the fastest, which ensures the fastest convergence of the integral.

The steepest descent path can be found numerically by calculating the tangent to the curve $L$ by taking the numerical derivative of $G(p ; \tau)=\mathrm{e}^{p t} \tilde{f}(p)$. Let $G(p ; \tau)=u(p ; \tau)+\mathrm{i} w(p ; \tau)$. We parametrize the integration curve by $x=x(\lambda), y=y(\lambda)$, for real $\lambda$. So $L^{\prime}=\{p=x(\lambda)+\mathrm{i} y(\lambda) \mid \lambda>0\}$. The curve is normalized by the requirement that

$$
\left(\frac{\mathrm{d} x}{\mathrm{~d} \lambda}\right)^{2}+\left(\frac{\mathrm{d} y}{\mathrm{~d} \lambda}\right)^{2}=1
$$

then define $\theta$ (counter-clockwise with respect to the positive $x$-axis) by

$\cos \theta=\frac{\mathrm{d} x}{\mathrm{~d} \lambda}, \quad \sin \theta=\frac{\mathrm{d} y}{\mathrm{~d} \lambda}$.

The change of $w$ along the curve can be represented by

$\frac{\mathrm{d} w}{\mathrm{~d} \theta}=w_{x} \cos \theta+w_{y} \sin \theta$,

which is required to be zero, so that $w=w\left(p_{1} ; \tau\right)$ remains zero along the integration curve. Then, by the CauchyRiemann equations, we have

$\frac{\mathrm{d} w}{\mathrm{~d} \lambda}=w_{x} \cos \theta+u_{x} \sin \theta=\operatorname{Im}\left(G^{\prime}\right) \cos \theta+\operatorname{Re}\left(G^{\prime}\right) \sin \theta=0$, 
So

$\theta=\arctan \left(-\frac{\operatorname{Im}\left(G^{\prime}\right)}{\operatorname{Re}\left(G^{\prime}\right)}\right)$

This value for $\theta$ gives the tangent to the integration curve. Therefore, once we know a point of the curve, say point 1 , the next point, point 2 will lie on the arc segment of a circle with center point 1 , with some radius $R$ and bounded by angles $\theta_{1}=\theta-\Delta \theta, \theta_{2}=\theta+\Delta \theta$. The derivative $G^{\prime}$ will be found through numerical differentiation, using an infinitesimal $\Delta p=\epsilon|p|$. The choices for $R, \Delta \theta$ and $\epsilon$ depend on the problem at hand.

\section{Application}

For the case with $F(r)=0$, we apply the three different numerical techniques as described above:

Method 1. Integration according to the analytical solution (third line in (5)), where we first determine the zeros of the integrand, then integrate from zero to zero and finally sum all contributions using the Euler summation technique (see among others van Wijngaarden [46] and the source text in Algol as Example 1, p. 447 in Naur [47].

Method 2. Application of the Laplace inversion technique by de Hoog et al. [30]. We used a MATLAB-script by Hollenbeck [48].

Method 3. Application of the steepest descent path through the complex p-plane as described in Sect. 6.

Method 1 is the most time consuming one. The other two methods require calculation times of the same order, but Method 2 turned out to be the fastest, since there is no need to find the steepest descent path. However, execution times could not be compared properly, since for Method 1 and 3 we have written FORTRAN-programs, while for Method 2 we use a MATLAB-script. Except for Method 2 we have full control on the relative and absolute precision of our results. For Method 2 we can modify some parameters to increase the accuracy, but there is not some kind of error criterium. All three methods applied to the examples below gave the same results up to the required precision.

\subsection{Constant boundary conditon}

Here, we shall treat the example of a Dirichlet boundary condition at the well $\left(\gamma^{\prime}=1, \delta^{\prime}=0\right.$, so $\gamma=1, \delta=0$ and thus also $\alpha=1, \beta=0$ ) with $F(r)=0, G(t)=G_{0}$, so $f(\rho)=0, g(\tau)=1$. We assume an injection, so we use the lower sign in the formulae, where appropriate. The function which has to be evaluated (see the third line of (5)) reads

$C(\rho, \tau)=\mathrm{e}^{\mp\left(\rho-\rho_{0}\right) / 2} \int_{0}^{\infty} \frac{1-\mathrm{e}^{-s \tau}}{s} \frac{A i(z) B_{0}\left(z_{0}\right)-B i(z) A_{0}\left(z_{0}\right)}{\pi\left(A_{0}^{2}\left(z_{0}\right)+B_{0}^{2}\left(z_{0}\right)\right)} \mathrm{d} s$.

Integrals of this type have the following properties. The first factor in the integrand is a slowly decreasing function, which behaves like $1 / s, s \rightarrow \infty$; the second one is an oscillating function of which the absolute size remains constant. Using the asymptotic behaviour of the Airy functions for large negative arguments one can find that the zeros of the second factor are approximatively $s_{n}=\frac{9}{4} n^{2} \pi^{2}\left(\rho^{3 / 2}-\rho_{0}^{3 / 2}\right)^{-2}$ for $n \rightarrow \infty$. For values of $\rho$ close to $\rho_{0}$ the zeros are far away from each other, while for large values of $\rho$ with respect to $\rho_{0}$ the zeros are lying close to each other. In that case one encounters easily loss of digits by adding up contributions for the integral of almost equal size but with opposite sign. It is clear that such an integrand gives rise to numerical problems. In Fig. 1a, the integrand has been shown for the values $\alpha=1, \beta=0, g(\tau)=1, \rho=8, \rho_{0}=1$, and $\tau=2$. Methods 2 and 3 give easily and quickly the same result $C(8,2)=1.006867846 \times 10^{-11}$, while Method 1 can not find more than five digits of accuracy (relative precision $1 \times 10^{-4}$ ), since the contributions from zero to the next zero of the integrand in the integral (52) are of the order of $1 \times 10^{-1}$, slowly decreasing to order $1 \times 10^{-2}$ for the $40^{\text {th }}$ term. That means 

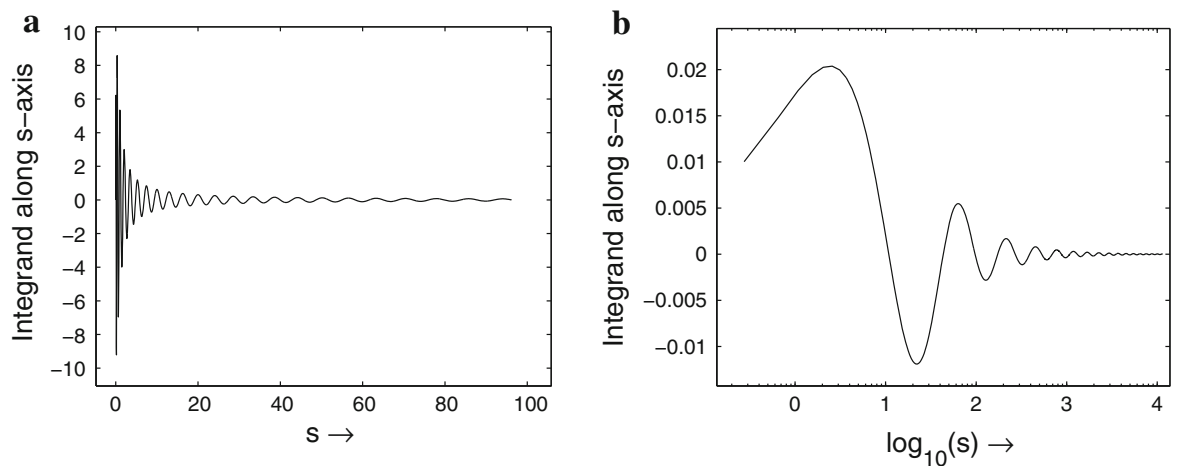

Fig. 1 a Integrand of the analytical representation (52) for the case $\alpha=1, \beta=0, \rho_{0}=1, \rho=8, \tau=2$. The factor in front of (52), $\exp \left(\left(\rho-\rho_{0}\right) / 2\right)$ is taken into account. This integrand is slowly decreasing and highly oscillatory. b Integrand of the analytical representation (52) for the case $\alpha=1, \beta=0, \rho_{0}=10, \rho=10.3, \tau=0.06$. The factor in front of $(52)$, $\exp \left(\left(\rho-\rho_{0}\right) / 2\right)$ is taken into account. The scaling of the horizontal axis is logarithmic to base 10 . This integrand is slowly decreasing and oscillating
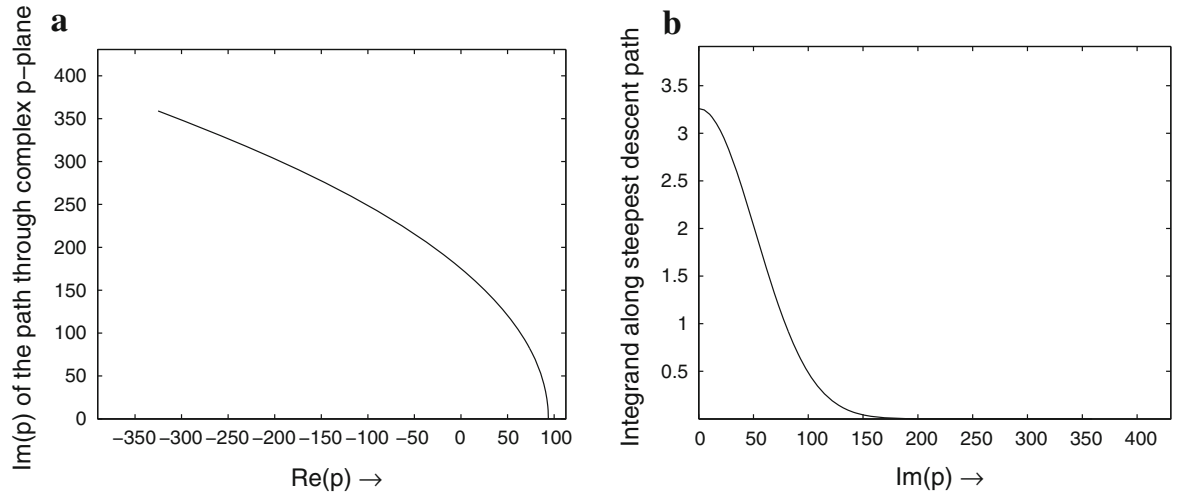

Fig. 2 a Steepest descent path through the complex $p$ plane to find the inverse of the Laplace transform for the case $\alpha=1, \beta=0, \rho_{0}=$ $10, \rho=10.3, \tau=0.06$. The path starts in the saddle point at $(93.792,0)$. b Integrand along the steepest descent path through the complex $p$ plane to find the inverse of the Laplace transform for the case $\alpha=1, \beta=0, \rho_{0}=10, \rho=10.3, \tau=0.06$. The integrand along this path is an exponentially decreasing function

that for $d$ digits of accuracy, the relative error in these contributions should be smaller than $1 \times 10^{-10-(d-1)}$, which gives $d \sim 5$ for the limits of the standard floating point system. For larger values of $\rho$, the performance of Method 1 becomes worse. In such cases one needs to apply Method 2 or Method 3.

Next we choose $\rho_{0}=10$. For $\tau=0.06$ and $\rho=10.3$ the integrand of (52) is shown in Fig. 1b, where the exponential factor $\mathrm{e}^{\mp\left(\rho-\rho_{0}\right) / 2}$ in front of this integral is taken into account; we show the steepest descent path through the complex $p$ plane (see Fig. 2a) and the corresponding integrand along that path (see Fig. 2b) is indeed an exponentially decreasing function, which is numerically favourable (see Sect. 6). For these particular parameters, the numerical result is $C(10.3,0.06)=6.6774197000 \times 10^{-3}$ for all the three numerical techniques (Methods 1 , 2 and 3) up to all the digits shown. We present graphs of $C(\rho, \tau)$ as a function of $\tau \in[0.01,2]$ for fixed $\rho=10.3$ (see Fig. 3a) and as a function of $\rho \in[10,12]$ for fixed $\tau=1$ (see Fig. 3b). These graphs have been obtained by Method 2. The other two methods gave the same results.

\subsection{Delta boundary condition}

Here, we take as boundary condition $g(\tau)=\delta(\tau)$, the Dirac delta function. All parameters are the same as for the second example in 7.1. We present graphs of $C(\rho, \tau)$ as a function of $\tau \in[0.01,2]$ for fixed $\rho=10.3$ (see Fig. 4a) and as a function of $\rho \in[10,12]$ for fixed $\tau=1$ (see Fig. 4b). Again, these graphs have been made by Method 2. 

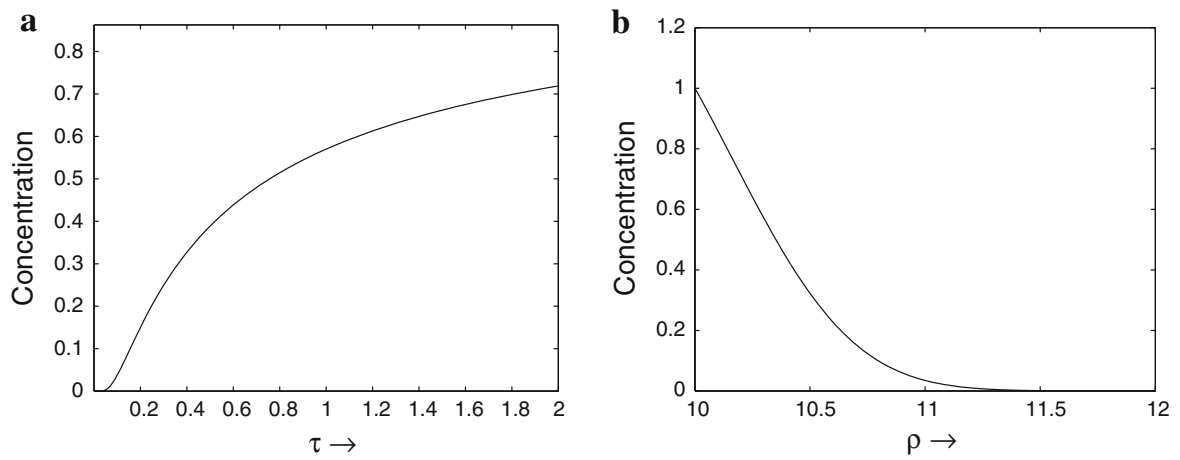

Fig. 3 a Example 7.1: concentration $C(\rho, \tau)$ as a function of $\tau$ for $g(\tau)=1, f(\rho)=0, \rho=10.3$, for the case $\gamma=\alpha=$ $1, \delta=\beta=0, \rho_{0}=10$. b Example 7.1: concentration $C(\rho, \tau)$ as a function of $\rho$ for $g(\tau)=1, f(\rho)=0, \tau=1$, for the case $\gamma=\alpha=1, \delta=\beta=0, \rho_{0}=10$
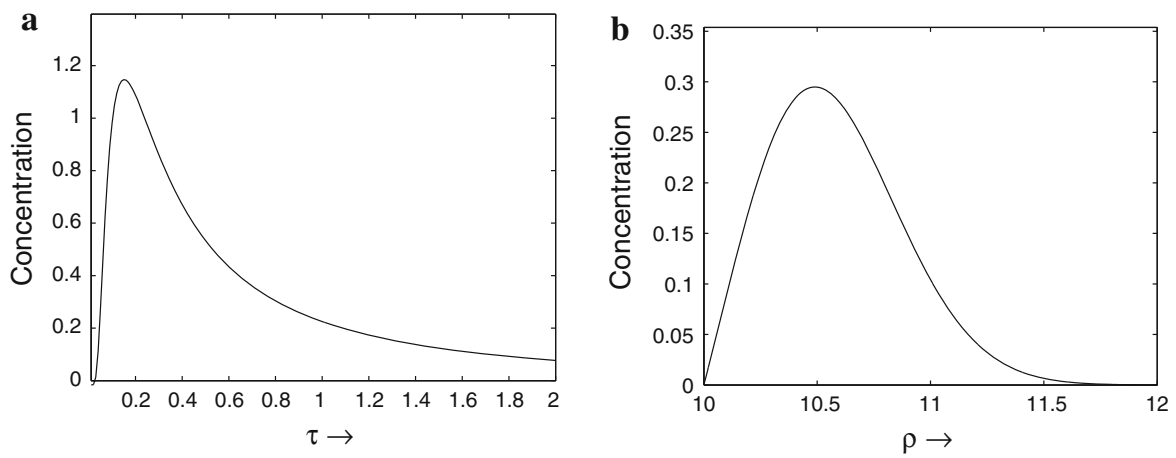

Fig. 4 a Example 7.2: concentration $C(\rho, \tau)$ as a function of $\tau$ for $g(\tau)=\delta(\tau), f(\rho)=0, \rho=10.3$, for the case $\gamma=\alpha=$ $1, \delta=\beta=0, \rho_{0}=10$. b Example 7.2: concentration $C(\rho, \tau)$ as a function of $\rho$ for $g(\tau)=\delta(\tau), f(\rho)=0, \tau=1$, for the case $\gamma=\alpha=1, \delta=\beta=0, \rho_{0}=10$
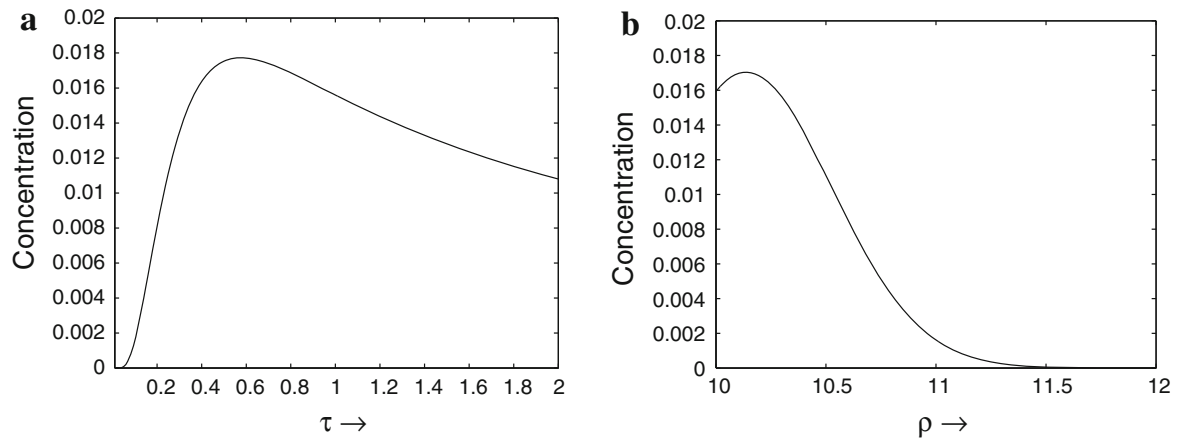

Fig. 5 a Example 7.3: concentration $C(\rho, \tau)$ as a function of $\tau$ for $g(\tau)=\exp \left(s_{0} \tau\right), s_{0}=-9, f(\rho)=0, \rho=10.3$, for the case $\gamma=1, \delta=-1(\operatorname{so} \alpha=1 / 2, \beta=-1), \rho_{0}=10$. b Example 7.3: concentration $C(\rho, \tau)$ as a function of $\rho$ for $g(\tau)=\exp \left(s_{0} \tau\right), s_{0}=$ $-9, f(\rho)=0, \tau=1$, for the case $\gamma=1, \delta=-1$ (so $\alpha=1 / 2, \beta=-1), \rho_{0}=10$. For $\rho=\rho_{0}=10$ the boundary condition $C-\partial C / \partial \rho=\exp (-9)$ has been satisfied

\subsection{Exponentially decreasing boundary condition}

As a final example, we apply a flux concentration boundary condition with an injection, so $\gamma^{\prime}=1, \delta^{\prime}=-a$, which gives $\gamma=1, \delta=-1$, and $\alpha=1 / 2, \beta=-1$, and we take as boundary condition $g(\tau)=\exp \left(s_{0} \tau\right)$, with $s_{0}=-9$. We present graphs of $C(\rho, \tau)$ as a function of $\tau \in[0.01,2]$ for fixed $\rho=10.3$ (see Fig. 5a) and as a function 
of $\rho \in[10,12]$ for fixed $\tau=1$ (see Fig. 5b). In Fig. 5b, the boundary condition $C-\partial C / \partial \rho=\exp (-9)$, for $\rho=\rho_{0}=10$ has been satisfied. This time, Fig. $5 \mathrm{a}$ and $\mathrm{b}$ have been obtained by Method 3 .

Acknowledgements The author thanks Cleve Moler (The Mathworks) and Matt Feinstein (Johns Hopkins University) for supplying information how to call exponentially scaled Airy functions from Matlab. This cannot be found in the standard documentation. The author is grateful to Kees Maas and Mark Bakker for suggestions on improving the presentation of this article.

Open Access This article is distributed under the terms of the Creative Commons Attribution Noncommercial License which permits any noncommercial use, distribution, and reproduction in any medium, provided the original author(s) and source are credited.

\section{Appendix A: Derivation of the Hankel transform}

In this Appendix, we outline the derivation of the Hankel transform. The theory for the construction of these transforms has been supplied by Naimark [36] (in $\S \$ 21.3,21.4,21.5$ ). First, we study the eigenvalue problem

$$
\begin{aligned}
& \frac{1}{\rho}\left(\frac{\partial^{2} f}{\partial \rho^{2}}-\frac{1}{4} f\right)=-s f, \quad \rho_{0}<\rho<\infty, \\
& \left.\left(\alpha f+\beta \frac{\partial f}{\partial \rho}\right)\right|_{\rho_{0}}=0 .
\end{aligned}
$$

A set of independent solutions is given by $\left\{D_{1}(\rho, s), D_{2}(\rho, s)\right\}$, see (16). By taking a linear combination of $D_{1}, D_{2}$, namely $u=D_{1}+\alpha / \beta D_{2}$, we arrive at a solution to (53). Like Naimark, we introduce the transform

$\phi_{N}(s)=T_{N}[f] \equiv \int_{\rho_{0}}^{\infty} \rho f(\rho) u(\rho, s) \mathrm{d} \rho$,

such that

$T_{N}\left[\frac{1}{\rho}\left(\frac{\partial^{2} f}{\partial \rho^{2}}-\frac{1}{4} f\right)\right]=-s T_{N}[f]$.

The back-transformation reads

$f(\rho)=T_{N}^{-1}\left[\phi_{N}\right]=\int_{0}^{\infty} \phi_{N}(s) \frac{1}{\pi} \operatorname{Im} M(s) \mathrm{d} s$,

where the coefficient $M(s)$ is a function of $s$ such that for $\operatorname{Im}(s)>0, v(\rho, s)+M(s) u(\rho, s) \in \mathcal{L}^{2}\left(\rho_{0}, \infty\right)$ as a function of $\rho$. The space $\mathcal{L}^{2}\left(\rho_{0}, \infty\right)$ contains all functions $f(\rho)$ defined on $\left(\rho_{0}, \infty\right)$ with $\int_{\rho_{0}}^{\infty}|f(\rho)|^{2} \mathrm{~d} \rho<\infty$. Here, the function $v$ equals $D_{2}$. To fulfill this requirement, we study the asymptotic behaviour of $D_{1}$ and $D_{2}$ (see formulae (10.4.60) and (10.4.64) in Abramowitz and Stegun [35])

$A i(z) \sim \pi^{-1 / 2}(-z)^{-1 / 4} \sin (\zeta-\pi / 4), \quad \zeta=\frac{2}{3}(-z)^{3 / 2}, \quad z \rightarrow \infty$,

$B i(z) \sim \pi^{-1 / 2}(-z)^{-1 / 4} \cos (\zeta-\pi / 4), \quad \zeta=\frac{2}{3}(-z)^{3 / 2}, \quad z \rightarrow \infty$.

For $\operatorname{Im}(s)>0$, we have

$z \sim-\rho s^{1 / 3}, \quad \rho \rightarrow \infty$,

$\zeta=\frac{2}{3}(-z)^{3 / 2} \sim \frac{2}{3} \rho^{3 / 2} s^{1 / 2}=\frac{2}{3} \rho^{3 / 2}(a+\mathrm{i} b), \quad b>0$,

and so

$\mathrm{e}^{-\mathrm{i} \zeta} \sim \mathrm{e}^{-\frac{2}{3} \rho^{3 / 2}(\mathrm{i} a-b)}=\mathrm{e}^{-\frac{2}{3} \rho^{3 / 2} a \mathrm{i}} \mathrm{e}^{\frac{2}{3} \rho^{3 / 2} b} \rightarrow \infty, \quad \rho \rightarrow \infty$. 
This means that the coefficient for $e^{-\mathrm{i} \zeta}$ in the asymptotic expansion of $u$ should be equal to zero, because the function $e^{-\mathrm{i} \zeta(\rho)} \notin \mathcal{L}^{2}\left(\rho_{0}, \infty\right)$. Using (57) and (58), we find

$$
\begin{aligned}
& A i(z) \sim \pi^{-1 / 2}\left(\rho s^{1 / 3}\right)^{-1 / 4}\left(\frac{-\mathrm{e}^{-\mathrm{i} \pi / 4}}{2 \mathrm{i}}\right) e^{-\mathrm{i} \zeta} \\
& =\pi^{-1 / 2}\left(\rho s^{1 / 3}\right)^{-1 / 4}\left(\frac{\mathrm{e}^{-\mathrm{i} \pi / 4}}{2}\right) \mathrm{ie}^{-\mathrm{i} \zeta} \equiv A\left(z_{\infty}\right) \mathrm{e}^{-\mathrm{i} \zeta}, \quad \rho \rightarrow \infty, \\
& B i(z) \sim \pi^{-1 / 2}\left(\rho s^{1 / 3}\right)^{-1 / 4}\left(\frac{\mathrm{e}^{-\mathrm{i} \pi / 4}}{2}\right) \mathrm{e}^{-\mathrm{i} \zeta} \equiv B\left(z_{\infty}\right) \mathrm{e}^{-\mathrm{i} \zeta}, \quad \rho \rightarrow \infty .
\end{aligned}
$$

That means that

$$
\begin{aligned}
& v+M u \sim\left\{-\pi s^{-1 / 3}\left(A\left(z_{\infty}\right) B i\left(z_{0}\right)-B\left(z_{\infty}\right) A i\left(z_{0}\right)\right)+M\left\{\pi\left(A\left(z_{\infty}\right) B i^{\prime}\left(z_{0}\right)-B\left(z_{\infty}\right) A i^{\prime}\left(z_{0}\right)\right)\right.\right. \\
& \left.\left.\quad+\alpha / \beta\left(-\pi s^{-1 / 3}\right)\left(A\left(z_{\infty}\right) B i\left(z_{0}\right)-B\left(z_{\infty}\right) A i\left(z_{0}\right)\right)\right\}\right\} \mathrm{e}^{-\mathrm{i} \zeta}, \quad \rho \rightarrow \infty .
\end{aligned}
$$

To fulfill the requirement for $\operatorname{Im}(s)>0, v+M u \in \mathcal{L}^{2}\left(\rho_{0}, \infty\right)$ we need that the factor for $e^{-\mathrm{i} \zeta}$ equals zero, and so, by the fact that $A\left(z_{\infty}\right) / B\left(z_{\infty}\right)=\mathrm{i}$,

$$
\begin{aligned}
M(s)= & {\left[\pi s^{-1 / 3}\left(A\left(z_{\infty}\right) B i\left(z_{0}\right)-B\left(z_{\infty}\right) A i\left(z_{0}\right)\right)\right] / } \\
= & \frac{\left[\pi\left(A\left(z_{\infty}\right) B i^{\prime}\left(z_{0}\right)-B\left(z_{\infty}\right) A i^{\prime}\left(z_{0}\right)\right)+\alpha / \beta\left(-\pi s^{-1 / 3}\left(A\left(z_{\infty}\right) B i\left(z_{0}\right)-B\left(z_{\infty}\right) A i\left(z_{0}\right)\right)\right)\right]}{\left(\mathrm{i} B i^{\prime}\left(z_{0}\right)-A i^{\prime}\left(z_{0}\right)\right)+\alpha / \beta\left(-s^{-1 / 3}\left(\mathrm{i} B i\left(z_{0}\right)-A i\left(z_{0}\right)\right)\right)} \\
= & -\frac{\beta\left(A i\left(z_{0}\right)-\mathrm{i} B i\left(z_{0}\right)\right)}{\alpha A i\left(z_{0}\right)-\beta s^{1 / 3} A i^{\prime}\left(z_{0}\right)-\mathrm{i}\left(\alpha B i\left(z_{0}\right)-\beta s^{1 / 3} B i^{\prime}\left(z_{0}\right)\right)} \\
= & -\frac{\beta\left(A i\left(z_{0}\right)-\mathrm{i} B i\left(z_{0}\right)\right)}{A_{0}\left(z_{0}\right)-\mathrm{i} B_{0}\left(z_{0}\right)} .
\end{aligned}
$$

See (6) for the definition of $A_{0}\left(z_{0}\right)$ and $B_{0}\left(z_{0}\right)$. Then

$$
\begin{aligned}
& \operatorname{Im} M(s)=\frac{\operatorname{Im}\left\{-\beta\left(A i\left(z_{0}\right)-\mathrm{i} B i\left(z_{0}\right)\right)\left(A_{0}\left(z_{0}\right)+\mathrm{i} B_{0}\left(z_{0}\right)\right)\right\}}{A_{0}^{2}\left(z_{0}\right)+B_{0}^{2}\left(z_{0}\right)}=\frac{-\beta A i\left(z_{0}\right) B_{0}\left(z_{0}\right)+\beta B i\left(z_{0}\right) A_{0}\left(z_{0}\right)}{A_{0}^{2}\left(z_{0}\right)+B_{0}^{2}\left(z_{0}\right)} \\
& =\frac{-\beta A i\left(z_{0}\right)\left(\alpha B i\left(z_{0}\right)-\beta s^{1 / 3} B i^{\prime}\left(z_{0}\right)\right)}{A_{0}^{2}\left(z_{0}\right)+B_{0}^{2}\left(z_{0}\right)}+\frac{\beta B i\left(z_{0}\right)\left(\alpha A i\left(z_{0}\right)-\beta s^{1 / 3} A i^{\prime}\left(z_{0}\right)\right)}{A_{0}^{2}\left(z_{0}\right)+B_{0}^{2}\left(z_{0}\right)}=\frac{\beta^{2} s^{1 / 3} / \pi}{A_{0}^{2}\left(z_{0}\right)+B_{0}^{2}\left(z_{0}\right)},
\end{aligned}
$$

so that

$f(\rho)=T_{N}^{-1}\left[\phi_{N}\right]=\int_{0}^{\infty} \phi_{N}(s) u(\rho, s) \frac{\beta^{2} s^{1 / 3}}{\pi^{2}\left(A_{0}^{2}\left(z_{0}\right)+B_{0}^{2}\left(z_{0}\right)\right)} \mathrm{d} s$.

If we compare (54) with (12), we see that (since $\left.u(\rho, s)=1 / \beta\left\{\beta D_{1}(\rho, s)+\alpha D_{2}(\rho, s)\right\}\right)$

$T_{N}[f]=\frac{1}{\beta} T[f], \quad$ and so $\phi_{N}(s)=\frac{1}{\beta} \phi(s)$,

and so

$f(\rho)=T^{-1}[\phi]=\frac{1}{\beta} T_{N}^{-1}[\phi]=\frac{1}{\beta} \int_{0}^{\infty} \phi(s) \frac{D_{1}(\rho, s)+\alpha / \beta D_{2}(\rho, s)}{\pi^{2}\left(A_{0}^{2}\left(z_{0}\right)+B_{0}^{2}\left(z_{0}\right)\right)} \beta^{2} s^{1 / 3} \mathrm{~d} s$.

This equals Eq. 14. 


\section{Appendix B: Hankel transform of $\mathrm{e}^{-\rho / 2}$ and $\rho \mathrm{e}^{-\rho / 2}$}

The evaluation of the integral $T\left[e^{-\rho / 2}\right]$ runs as follows.

$$
\begin{aligned}
T & {\left[e^{-\rho / 2}\right]=\int_{\rho_{0}}^{\infty} \rho^{\prime} \mathrm{e}^{-\rho^{\prime} / 2}\left\{\beta D_{1}(\rho, s)+\alpha D_{2}(\rho, s)\right\} \mathrm{d} \rho^{\prime} } \\
= & \int_{\rho_{0}}^{\infty} \rho^{\prime} \mathrm{e}^{-\rho^{\prime} / 2}\left(-\pi s^{-1 / 3}\right)\left\{A i\left(z^{\prime}\right) B_{0}\left(z_{0}\right)-B i\left(z^{\prime}\right) A_{0}\left(z_{0}\right)\right\} \mathrm{d} \rho^{\prime} \\
= & \int_{z_{0}}^{-\infty}\left(\frac{1}{4} s^{-1}-z^{\prime} s^{-1 / 3}\right) \mathrm{e}^{-1 /(8 s)+1 /\left(2 s^{1 / 3}\right) z^{\prime}} \pi s^{-2 / 3}\left\{A i\left(z^{\prime}\right) B_{0}\left(z_{0}\right)-B i\left(z^{\prime}\right) A_{0}\left(z_{0}\right)\right\} \mathrm{d} z^{\prime} \\
= & \pi s^{-2 / 3} \mathrm{e}^{-1 /(8 s)}\left\{\frac{1}{4} s^{-1} J_{0}-s^{-1 / 3} J_{1}\right\},
\end{aligned}
$$

with, $i=0,1,2$,

$J_{i}=\int_{z_{0}}^{-\infty}\left(z^{\prime}\right)^{i} \mathrm{e}^{\theta z^{\prime}}\left\{A i\left(z^{\prime}\right) B_{0}\left(z_{0}\right)-B i\left(z^{\prime}\right) A_{0}\left(z_{0}\right)\right\} \mathrm{d} z^{\prime}, \theta=\frac{1}{2} s^{-1 / 3}$.

Integration by parts gives for $J_{1}$

$J_{1}=\mathrm{e}^{\theta z_{0}} \frac{\left(\alpha-\frac{1}{2} \beta\right)}{\pi}+\theta^{2} J_{0}$.

Insertion of (70) into (68) gives

$T\left[\mathrm{e}^{-\rho / 2}\right]=-s^{-1} \mathrm{e}^{-\rho_{0} / 2}\left(\alpha-\frac{1}{2} \beta\right)$.

Along the same lines it is possible to derive

$T\left[\rho \mathrm{e}^{-\rho / 2}\right]=\pi s^{-2 / 3} \mathrm{e}^{-1 /(8 s)}\left\{\frac{1}{16} s^{-2} J_{0}-\frac{1}{2} s^{-4 / 3} J_{1}+s^{-2 / 3} J_{2}\right\}$.

Integration by parts gives for $J_{2}$

$J_{2}=\left(z_{0}+\frac{1}{4} s^{-2 / 3}\right) \mathrm{e}^{\theta z_{0}} \frac{\left(\alpha-\frac{1}{2} \beta\right)}{\pi}-\frac{\beta}{\pi} s^{1 / 3} \mathrm{e}^{\theta z_{0}}+2 \theta J_{0}+\theta^{4} J_{0}$,

where we used (70). Since we need to know $T\left[\rho \mathrm{e}^{-\rho / 2}\right]$ only if $\alpha=\frac{1}{2} \beta$, we find by substituting (70) and (73) into (72)

$T\left[\rho \mathrm{e}^{-\rho / 2}\right]=-s^{-1} \mathrm{e}^{-\rho_{0} / 2} \beta+\pi s^{-2 / 3} \mathrm{e}^{-1 /(8 s)} s^{-1} J_{0}$.

Evaluation of $T\left[\frac{1}{\rho} s^{-1} \mathrm{e}^{-\rho / 2}\right]$ is easy and gives

$T\left[\frac{1}{\rho} s^{-1} \mathrm{e}^{-\rho / 2}\right]=\pi s^{-2 / 3} \mathrm{e}^{-1 /(8 s)} s^{-1} J_{0}$.

This means that

$T\left[\rho \mathrm{e}^{-\rho / 2}-\frac{1}{\rho} s^{-1} \mathrm{e}^{-\rho / 2}\right]=-s^{-1} \mathrm{e}^{-\rho_{0} / 2} \beta$. 


\section{References}

1. Hoopes JA, Harleman DRF (1967) Dispersion in radial flow from a recharge well. J Geophys Res 72(14):3595-3607

2. Dagan G (1971) Perturbation solutions of the dispersion equation in porous mediums. Water Resour Res 7(1):135-142

3. Gelhar LW, Collins MA (1971) General analysis of longitudinal dispersion in nonuniform flow. Water Resour Res 7(4):1511-1521

4. Bear J (1972) Dynamics of fluids in porous media. American Elsevier, New York

5. Bachmat Y, Mandel S, Bugayevski M (1988) A single-well tracer technique for evaluating aquifer parameters, I. Theoretical work. J Hydrol 99(1-2):143-163

6. Ogata A (1958) Dispersion in porous media. Ph.D. dissertation, Northwestern University, Evanston.

7. Tang DH, Babu DK (1979) Analytical solution of a velocity dependent dispersion problem. Water Resour Res 15(6):1471-1478

8. Moench AF, Ogata A (1981) A numerical inversion of the Laplace transform solution to radial dispersion in a porous medium. Water Resour Res 17(1):250-252

9. Hsieh PA (1986) A new formula for the analytical solution of the radial dispersion problem. Water Resour Res 22(11):1597-1605

10. Chen CS (1987) Analytical solutions for radial dispersion with Cauchy boundary at injection well. Water Resour Res 23(7):12171224

11. Chen CS, Woodside GD (1988) Analytical solution for aquifer decontamination by pumping. Water Resour Res 24(8):1329-1338

12. Chen JS, Liu CW, Chen CS, Yeh HD (1996) A Laplace transform solution for tracer tests in a radially convergent flow field with upstream dispersion. J Hydrol 183(3-4):263-275

13. Bruggeman G (1999) Analytical solutions of geohydrological problems. Developments in water science, nr. 46. Elsevier, Amsterdam

14. Huang J, Christ JA, Goltz MN (2010) Analytical solutions for efficient interpretation of single-well push-pull tracer tests. Water Resour Res 46(8):W08538

15. Chen CS (1985) Analytical and approximative solutions to radial dispersion from an injection well to a geological unit with simultaneous diffusion into adjacent strata. Water Resour Res 21(8):1069-1076

16. Chen CS (1986) Solutions for radionuclide transport from an injection well into a single fracture in a porous formation. Water Resour Res 22(4):508-518

17. Valocchi AJ (1986) Effect of radial flow on deviations from local equilibrium during sorbing solute transport through homogeneous soils. Water Resour Res 22(12):1693-1701

18. Moench AF (1989) Convergent radial dispersion: a Laplace transform solution for aquifer tracer testing. Water Resour Res 25(3):439-447

19. Yates SR (1990) An analytical solution for one-dimensional transport in heterogeneous porous media. Water Resour Res 26(10):2331-2338

20. Goltz MN, Oxley ME (1991) Analytical modeling of aquifer decontaminating by pumping when transport is affected by rate-limited sorption. Water Resour Res 27(4):547-556

21. Moench AF (1991) Convergent radial dispersion: a note on evaluation of the Laplace transform solution. Water Resour Res 27(12):3261-3264

22. Chen JS, Chen CS, Chen CY (2007) Analysis of solute transport in a divergent flow tracer test with scale-dependent dispersion. Hydrol Process 21(18):2526-2536

23. Chen JS, Liang CP, Chen CY, Liu CW (2007) Composite analytical solutions for a soil vapour extraction system. Hydrol Process 21(11):1506-1516

24. Chen JS (2010) Analytical model for fully three-dimensional radial dispersion in a finite-thickness aquifer. Hydrol Process 24(7):934-945

25. Chen JS, Liu CW, Liao CM (2002) A novel analytical power series solution for solute transport in a radially convergent flow field. J Hydrol 266(1-2):120-138

26. Chen JS, Liu CW, Liao CM (2003) Two-dimensional Laplace-transformed power series solution for solute transport in a radially convergent flow field. Adv Water Resour 26(10):1113-1124

27. Leijnse A, Veling EJM, Beneš V, Kopáčková M (1987) Determination of longitudinal dispersivity by the single well tracer test. In: Vrba J, Švoma J (eds) Proceedings: symposium on ground-water protection areas, Karlovy Vary Congress, Czechoslovakia 1986, no. XIX, Part 2 in Mémoires. International Association of Hydrogeologists, "Novinář” Publishing House, Prague, pp 455-466

28. Beneš V, Kopáčková M, Tvrdý M (1985) Mathematical solution of the transport equation for determining longitudinal hydrodynamic dispersivity using the single well tracer pulse method. Technical report. Stavební geologie n.p., and Matematický ústav ČSAV, Praha, Czechoslovakia

29. Veling E (2001) Analytical solution and numerical evaluation of the radial symmetric convection-diffusion equation with arbitrary initial and boundary data. In: Gehrels H, Peters NE, Hoehn E, Jensen K, Leibundgut C, Griffioen J, Webb B, Zaadnoordijk WJ, (eds) Impact of human activity on groundwater dynamics, IAHS Publ. no. 269 of IAHS series of proceedings and reports. Centre for Ecology and Hydrology, Wallingford, UK. Proceedings of a symposium held during the sixth IAHS scientific assembly at Maastricht, The Netherlands. IAHS Press, Wallingford, pp 271-276

30. de Hoog FR, Knight JH, Stokes AN (1982) An improved method for numerical inversion of Laplace transforms. SIAM J Sci Stat Comput 3(3):357-366

31. Talbot A (1979) The accurate numerical inversion of Laplace transforms. J Inst Math Appl 23:97-120

32. Evans GA (1993) Numerical inversion of Laplace transforms using contour methods. Int J Comput Math 49:93-105 
33. Evans GA, Chung KC (2000) Laplace transform inversions using optimal contours in the complex plane. Int J Comput Math 73:531-543

34. Kreft A, Zuber A (1978) On the physical meaning of the dispersion equation and its solution for different initial and boundary conditions. Chem Eng Sci 33:1471-1480

35. Abramowitz M, Stegun IA (eds) (1964) Handbook of mathematical functions. National Bureau of Standards, Washington, DC. http://www.math.hkbu.edu.hk/support/aands/toc.htm

36. Naimark MA (1968) Linear differential operators, part II. Linear differential operators in Hilbert space. Frederick Ungar Publishing Co., New York

37. Stehfest H (1970) Algorithm 368. Numerical inversion of Laplace transforms. Commun ACM 13:47-49; Erratum 13:624

38. Abate J, Whitt W (2006) A unified framework for numerically inverting Laplace transforms. INFORMS J Comput 18(4):408-421

39. Avdis E, Whitt W (2007) Power algorithms for inverting Laplace transforms. INFORMS J Comput 19(3):341-355

40. Wong R (1989) Asymptotic approximations of integrals. Academic Press, Inc., San Diego

41. Amos DE (1986) Algorithm 644: a portable package for Bessel functions of a complex argument and nonnegative order. ACM Trans Math Softw 12:265-273

42. Amos DE (1990) Remark on algorithm 644. ACM Trans Math Softw 16(4):404

43. Amos DE (1995) A remark on algorithm 644: a portable package for Bessel functions of a complex argument and nonnegative order. ACM Trans Math Softw 21(4):388-393

44. Gordon RG (1969) New method for constructing wavefunctions for bound states and scattering. J Chem Phys 51(1):14-25

45. Schulten Z, Anderson DGM, Gordon RG (1979) An algorithm for the evaluation of the complex Airy function. J Comput Phys 31:60-75

46. van Wijngaarden A (1953) A transformation of formal series. Indag Math 15:522-543

47. Naur P (1963) Revised report on the algorithmic language Algol 60. Numer Math 4:420-453

48. Hollenbeck KJ (1998) INVLAP.M: a Matlab function for numerical inversion of Laplace transforms by the de Hoog algorithm. Algorithm contributed to the Matlab community 